\title{
Composite Materials for Some Radiophysics Applications
}

\author{
S.B.Bibikov ${ }^{1}$ and M.V. Prokof'ev² \\ ${ }^{1}$ Emanuel Institute of Biochemical Physics (Russ.Acad.Sci.) \\ ${ }^{2}$ Moscow Aviation Institute (Technical State University) \\ 1,2SPE "Radiostrim" Ltd \\ Russian Federation
}

\section{Introduction}

The development of modern radio engineering can be characterized by an increase in carrier frequencies in the gigahertz range, as well as the introduction of new systems of ultrafast and ultrawideband signals. These applications require the use of the ultrawideband microwave absorbers and radio shielding materials for the equipment functionality, radioelectronic equipment compatibility, electromagnetic safety, test of the equipment. These materials must possess not only specific radio physical, but also necessary exploitational properties.

Depending on the tasks and requirements there are different approaches to design of the radioabsorbing materials. First of all, the materials which interact with non-ionization electromagnetic irradiation (EMI) can be sorted in two wide classes: the one is the shielding materials and the second is the materials absorbing EMI.

As far as materials for electromagnetic shielding, the principle of their work is simple enough. High conductivity (sometimes - magnetic permeability) provides high screening coefficient. Quit different situation is in the problem of absorbance of the electromagnetic irradiation. Finally, it is necessary that energy of electromagnetic wave should be converted to heat of the material. But substances able to absorb electromagnetic irradiation and process highest conductivity (e.g. metals) provide too high reflections of electromagnetic waves due to induced currents. To solve this contradiction, some special techniques are applied.

It is necessary to keep in mind that there are many requirements to the material. Namely the composite materials are convenient for satisfaction of a number of the necessary parameters. Commonly, a composite material consists of a matrix, usually - polymer, which determines mainly mechanical properties of a material, and a filler - dispersed phase, which provides specific electrical and magnetic properties and therefore radiotechnical parameters of a composite in whole. Different media are using as the matrix or a substance: - Si-O- based polymers, thermoplastics, porous polymer materials, etc.

To reduce the reflection from the interface between the resistance medium and the free space, the constructions with the volume resistance that is decreasing in the direction to inside are used. That's why it is necessary to know the rules which determine the resistivity of a composite material and to use the technology which guarantees homogeneous material with exactly predicted volumetric resistance. Besides, it is necessary to take in account a frequency 
dispersion of a electrical properties. Electrical properties of the composite materials in DC regime are determined by a percolation theory, and the properties in radiofrequency region strongly depend on geometry of the filler and are described by a polarization theory.

Another way to avoid unnecessary reflections from the materials placed on a reflective substrate is the using of a ferromagnetic phase. In this case so-called "wave resistance" of the media is match better to the free space wave resistance. To expand frequency region of effective absorbance down, the higher magnetic permeability of the composite materials should be achieved. For example, interesting results have been received, when there was a crystallization of an amorphous magnetic alloy.

\section{Resistive electroconducting composite}

The development of modern radioelectronics implies both the development of new and modernization of existing measuring instruments, and new challenges associated with the problems of electromagnetic compatibility. These problems cannot be solved without application of ultrawideband radio shielding and radio absorbing materials in radio engineering systems, the equipment and test benches.

At present theoretical principles are in detail developed and practical technologies of reception of microwave absorbers for various regions of a radio spectrum are mastered. The problem of wideband absorbers for frequencies $10^{6} \ldots 10^{11} \mathrm{~Hz}$ is still very relevant in spite of recently conducted studies of materials based on nanostructured composite thin film structures (see, e.g., [1]). For example, the non-reflective material for the walls of an anechoic chamber sometimes can provide the frequency range from $\sim 100 \mathrm{MHz}$ up to $100 \mathrm{GHz}$. It is not impossible to use ferrite-like material in this region because of magnetic permeability dispersion. But it is real to build the effective absorber with sizes of the order of a highest wavelength $\lambda$ based on generally-dielectric losses.

Practice shows that the approach involving the use of resistive materials for ultra-wideband applications (Gorbatenko \& Bibikov, 2006; Titov et al., 2008) will be very useful because of its adaptability, flexibility of implementation and cost effectiveness. Also, the development of radio frequency absorbers for low-frequency range-based approach uses of high magnetic permeability, has not yet been exhausted, and requires further study.

Let's consider one of the widely used approaches to obtaining microwave absorbers based on materials with Joule losses, or the so-called bulk-resistive materials. One of the advantages of this approach is the adaptability to obtain electrically conductive composite materials and the possibility of building structures on the basis of gradient materials with different concentrations of the conducting phase. Of course, one of the tasks, which must be solved with the construction of the radio absorbing materials, is the account of the dispersion of dielectric permittivity and magnetic permeability.

Commonly, the radio physical properties of the composite materials used in a practice are determined by the next:

- Conductivity of the filler;

- Concentration and conditions of the dispersion of the filler;

- Shape of the filler;

- Permittivity and electrical disruptive strength of the matrix.

It is known that the range of the bulk conductivity of composite material effectively absorbing microwave irradiation lies in the range $10^{-1} \div 10^{1} \Omega^{-1} \mathrm{~m}^{-1}$ (Gorshenev et al, 1997). To reach these values it is necessary to use the fillers possessing high conductivity, e.g. of metal or carbon type. 
Material of the matrix mainly provides physical and mechanical properties. Usually, polymeric materials are used as the matrices, these are: thermoplastics (for example, polyethylene, polyvinyl chloride, polyurethane, etc.), elastoplastics (silicon materials, etc.).

For the good formability of the material is sometimes necessary to use a plasticizer. For example, to improve the mechanical properties of PVC, the dioctylphthalate is used in an amount from 20 to $60 \%$ weight. Different components can be used for an improvement in the quality of composite materials, but in contrast to the functional filler and the basic component of matrix, they do not substantially influence the radio physical properties of the materials.

It is important to remember that the composite materials, in contrast to the solutions and the plasticized polymers, are not mixtures at the molecular level. The sizes of the particles of the filler always exceed the molecular dimensions. Even in the case of graphene or nanotubes used as the filler, the longitudinal particle sizes are much larger than the distance C-C bond. According to the nature of the distribution of components, the composite materials can be divided into the following classes:

- Materials constitute the layered structure

- Materials containing fiberfill;

- Matrix mixtures;

- Statistical mixtures.

The first two are clear from the material structure determination.

In the matrix polymer mixture forms a continuous medium in which discretely distributed filler particles do not come into contact with each other. In this case, the phases are unequal mixture.

Statistical mixtures are characterized by the disordered distribution of polymer and filler, and both phases are equal.

\subsection{Basis of the percolation theory}

One of key parameters determining radiophysical properties of the composite is its effective conductivity, which depends on properties of the conductive filler and conditions of its distribution in the matrix. Determination of the value of effective conductivity - is the global problem in the theory of heterogeneous media, in particular, percolation theory.

To calculate the effective conductivity without the frequency dependence of the inhomogeneous system is necessary to solve the system of equations

$$
\operatorname{div} \mathbf{j}=0, \operatorname{rot} \mathbf{E}=0
$$

under appropriate boundary conditions. Here E - electric field vector, $\mathbf{j}$ - current density vector. Effective conductivity is determined as

$$
\langle\mathbf{j}\rangle=\sigma_{e}\langle\mathbf{E}\rangle \text {. }
$$

There $<\ldots>$ means averaging over the volume $V$ of the sample. Then we assume $V \rightarrow \infty$. Following the theory, the effective conductivity of the system can be written as

$$
\sigma_{e}\left(p ; \sigma_{1}, \sigma_{2}\right)=\sigma_{1} \cdot f(p, h)
$$

where $h=\sigma 2 / \sigma 1$ - the ratio of conductivities of the phases, $p$ - volume concentration of the first component. The function $\mathrm{f}(\mathrm{p}, \mathrm{h})$ - "dimensionless conductivity" - plays a fundamental role in the whole theory of transport in isotropic two-component media. It is possible to find 
effective conductivity for any concentration of filler with $h \sim 1$ or for arbitrary $h$ with $p \ll 1$. In general, the problem of calculating the conductivity of disordered composites with arbitrary values of $p$ and $h$ has no analytical solution. To solve this problem, in the theory of heterogeneous structures, so-called "approximation of effective medium" has been proposed, or EMA (Kirkpatrick, 1973). Analysis shows that the system with the very different conductivities of components undergoes phase transition of the type metaldielectric with the specific relationship of concentrations. According to percolation theory, the conductivity of the system in the region of the phase transition is described by the following asymptotics (Efros \& Shklovskii, 1976):

$$
\begin{aligned}
& \tau>0, \Delta_{0} \ll \tau \ll 1: f=\tau^{t}\left\{A_{0}+A_{1}\left(\frac{h}{\tau^{t / s}}\right)+A_{2}\left(\frac{h}{\tau^{t / s}}\right)^{2}+\ldots\right\} \\
& |\tau| \ll \Delta_{0}: \quad f=h^{s}\left\{a_{0}+a_{1}\left(\frac{h}{\tau^{t / s}}\right)+a_{2}\left(\frac{h}{\tau^{t / s}}\right)^{2}+\ldots\right\} \\
& \tau<0, \Delta_{0} \ll|\tau| \ll 1: f=\frac{h}{(-\tau)^{q}}\left\{B_{1}+B_{2}\left(\frac{h}{(-\tau)^{t / s}}\right)+\ldots\right\}
\end{aligned}
$$

where $\tau=\left(p-p_{\mathrm{c}}\right) / p_{\mathrm{c}}, p$ - critical concentration; $\Delta_{0}=h^{\mathrm{s} / \mathrm{t}}$ - the size of the "smearing", and $t, \mathrm{~s}$ and $q$ - critical indexes, which are connected by the relation:

$$
t / s=t+q
$$

The values of indices depend on the dimensionality of the system:

- $\quad$ for the two-dimensional case $\mathrm{t}=\mathrm{q} \approx 1.3$ and $\mathrm{s}=0.5$ (exactly);

- $\quad$ for the three-dimensional case $t \approx 2 ; \mathrm{q} \approx 0.8$ and $\mathrm{s} \approx 0.7$ (exactly).

The problem of conductivity of composites with periodic structure is simpler. Here it is sufficient to find the potential within a single cell. Following (Stauffer, 2003), we assume for the calculations that the percolation threshold $p_{c}=0.31$ for the three-dimensional case of a cubic lattice. In this case we can write a direct expression for the conductivity of the composite on the concentration of inclusions in the case of $3 \mathrm{~d}$ :

$$
\sigma=\sigma_{1}\left(p-p_{c}\right)^{2 v}
$$

where $v=0.9$ for three-dimensional case.

\subsection{Type of conduction fillers}

If the composite material is used as microwave absorber or shielding, the basic functional characteristics are determined by the radiophysical properties of the filler, which should ensure effective action presents the interaction with the applied field and the dissipation of electromagnetic energy. It is necessary that the filler has at least one of the following qualities:

- Ensure that the macroscopic bulk or surface currents in the material;

- Creation of a generalized dielectric loss;

- Increasing the magnetic losses;

- $\quad$ Increasing the effective permeability of the composite. 
For this purposes, the next materials are used as the fillers:

- Materials on the basis of graphite-like structures - carbon black, carbon fibers, nanotubes, nanodisperse forms of carbon,

- Powders of metals and alloys, both in polycrystalline and amorphous state, providing electrical composite and sometimes necessary magnetic properties,

- Ferromagnetic materials the basis of iron powders,

- Conducting non-metallic materials - conductive polymers, systems with conjugated bonds (polyaniline, polypyrrole, polydiacetylene, etc.).

Commonly, the fillers based on various forms of graphite, are used. This is due to the fact that firstly, the graphite has a conductivity that lower by about 2 orders of magnitude then for metals, secondly, a more chemically inert and therefore less prone to corrosion than the metals, and in the third, it can easily be used in a variety of geometric shapes - spherical or disk-shaped particles and fibers.

Valuable feature of some kinds of carbon blacks is their ability to structured into chains. Structure and properties of different carbon blacks have a decisive importance on the properties of electromagnetic radiation shielding and absorbing materials, so you should always take into account the types of technology and preparation of various carbon blacks and graphite.

\subsection{The influence of the geometry of the filler}

The electrical and radiophysics properties of composite substantially depend on the geometry of filler. First of all, the discussion deals with the ratio of length to the transverse size of the inclusions. The task of determining the electrophysical parameters of the composite on the basis of the data about electrical conductivity and form of filler is no way trivial, to this the significant number of works is devoted.

Detail theoretical study of the electrophysical properties of composites containing elongated conducting inclusions - sticks - is presented in a work (Lagarkov \& Sarychev, 1996). The effective-medium approximation was developed in this work and the equation was derived to calculate an effective dielectric constant of the composites in the quasistatic case and for the high frequency. The theory predicts very large values of the effective dielectric constant in a wide range of the stick concentration. There was found that the dielectric constant can exhibit various dispersive behaviors. It can has relaxation behavior, power-law scaling behavior, or resonance dependence on the frequency. The resonance dependence occurs when the skin effect is strong and wavelength is comparable to the stick length. The diffraction of electromagnetic waves on a conducting stick - is a problem of classical electrodynamics, so one has to solve Maxwell's equations to find the polarizability for a particle in the composite illuminated by an electromagnetic wave. The particle is supposed to be embedded in the "effective medium" with conductivity. Then the effective conductivity $\sigma_{\mathrm{e}}$ is determined by the condition that the averaged polarizability of all particles shall vanish. In the condition of for the aspect ratio of the filler: $a / b \ll 1(a-$ transverse, $\mathrm{b}$ - longitudinal dimension), there was derived the following expression for the effective complex dielectric permittivity $\varepsilon$ e of the composite:

$$
\varepsilon_{e}=\varepsilon_{d} \cdot \frac{p}{p_{c}} \cdot \frac{a}{b \cdot \ln (a / b)} \cdot\left[\cos \left(\sqrt{\varepsilon_{d}} a k\right)-i \varepsilon_{d}(a k)^{2} \cdot \frac{\ln (a / b)}{2}\right]
$$

where $\varepsilon_{\mathrm{d}}$ - the complex permittivity of the conducting inclusions, $k$ - wave vector. 
If the composite is a statistical mixture of interacting components, there are dependences for the effective permittivity of the material (with a limited difference of complex values $\varepsilon$ ). General formulas for calculating the $\varepsilon_{e}$ have the form

$$
\begin{gathered}
\varepsilon_{e}=\varepsilon_{2}+\left(\varepsilon_{1}-\varepsilon_{2}\right) p_{1} K_{1} \\
\left(\varepsilon-\varepsilon_{1}\right) p_{1} K_{1}+\left(\varepsilon-\varepsilon_{2}\right) p_{2} K_{2}=0
\end{gathered}
$$

where $K_{1}$ and $K_{2}$ - ratio of the average electric field strengths in the components to the average field strength in the composite. Exact solution of equation (1) is possible only for two cases: for a layered dielectric when the electric field vector parallel to 1 - the interfaces between the planes and 2 - the axes of the cylinders, uniaxially oriented environment. In these cases $K_{1}=1$, hence $\varepsilon_{e}=p_{1} \varepsilon_{1}+p_{2} \varepsilon_{2}$. In other cases, for calculation of $K_{1}$ and $K_{2}$ need to make assumptions about the permeability of the medium $\varepsilon^{*}$. The assumption that $\varepsilon^{*}=\varepsilon_{2}$ corresponds to the matrix mixture, and the assumption that $\varepsilon^{*}=\varepsilon_{e}$ means equality of the components and corresponds to the condition of the statistical mixture. Thus, for the mixtures of $\mathrm{n}$ components, the following approximate expressions, or the so-called formulas of mixing are exist:

$$
\varepsilon_{e}^{x}=\sum_{i=1}^{n} p_{i} \cdot \varepsilon_{i}^{x}
$$

where $p_{\mathrm{i}}$ - volume fraction of the $\mathrm{i}$-th component, $\mathrm{x}$ - a value characterizing the distribution of the components. In particular, for a system in which the components are connected in parallel (layered plastics), we put $x=+1$; similarly, for a system in which the components are connected in series, we put $x=-1$. Often the case when the components are distributed randomly. Then, after substituting $x=0$ and differentiating expression (8), we obtain a formula, often used in engineering practice:

$$
\begin{aligned}
& \ln \left(\varepsilon_{e}\right)=p_{1} \cdot \ln \left(\varepsilon_{1}\right)+p_{2} \cdot \ln \left(\varepsilon_{2}\right)+\ldots+p_{n} \cdot \ln \left(\varepsilon_{n}\right), \\
& \left(p_{1}+p_{2}+\ldots+p_{n}=1\right)
\end{aligned}
$$

Note that these expressions are used for both real and imaginary part of permittivity.

For the special case of matrix mixtures with spherical inclusions, we can write the next equation:

$$
\frac{\varepsilon_{e}-\varepsilon_{\mathrm{m}}}{\varepsilon_{e}+\varepsilon_{\mathrm{m}}}=\sum_{i} p_{i} \frac{\varepsilon_{i}-\varepsilon_{\mathrm{m}}}{\varepsilon_{i}+2 \varepsilon_{\mathrm{m}}}
$$

where $\varepsilon_{\mathrm{m}}$ - permeability of the matrix, $\varepsilon_{i}$ - permeability of the components. Similar, for the statistical multicomponent mixtures:

$$
\sum_{i} p_{i} \frac{\varepsilon_{i}-\varepsilon_{e}}{\varepsilon_{i}+2 \varepsilon_{e}}=0
$$

From expression (7) it is possible to obtain the frequency dispersion of dielectric constant for the elongated inclusions. Analysis shows that in the high-frequency region in this case the composite is characterized by the resonance type of dispersion $\varepsilon$. 
Many composite materials, both traditional and with nanoscale inclusions are characterized by the frequency dispersion of permeability, which includes the relaxation part, which is similar to the dipole-dipole relaxation of Debye:

$$
\varepsilon_{e}=\varepsilon_{\infty}+\frac{\varepsilon_{c}-\varepsilon_{\infty}}{1+(i \omega \tau)^{1-\alpha}}
$$

where $\varepsilon c$ and $\varepsilon \infty$ - the dielectric constant at low and high frequency limits, respectively, $\tau$ time of relaxation, and a dimensionless exponent $(1-\alpha)$ characterizes the distribution of relaxation times, at $a=0$ there is no smearing of the relaxation times. Let us note that the same in the form expression (with $[\mathrm{f}]=0$ ) is correct for the structure of heterogeneous layered composite, this so-called polarization of Maxwell-Wagner type. The next section shows an example of analyzing the frequency dispersion of dielectric composite material used in practice.

\subsection{Porous electroconducting materials}

It is possible to consider porous material with a large specific surface area, covered with the thin electroconducting film, as a variety of the composite material, which is characterized by high effective electrical conductivity with the insignificant portion of filler. Such material can be, for example, polyurethane foam, whose pores are covered with graphite film.

Fig.1 presents the material consists of polyurethane foam (PUF) with the ultra-dispersed carbonic filler, introduced into the system in an amount providing the dc conductivity. This composite material based on the polyurethane foam is a foamed structure with open pores coated with ultrafine graphite composition. Structure of the polyurethane can be characterized by a number of parameters that affect the electrical properties of the material: density, linear dimensions of cells, their degree of elongation and orientation relative to the direction of foaming, the degree of connectedness, wall thickness, etc.

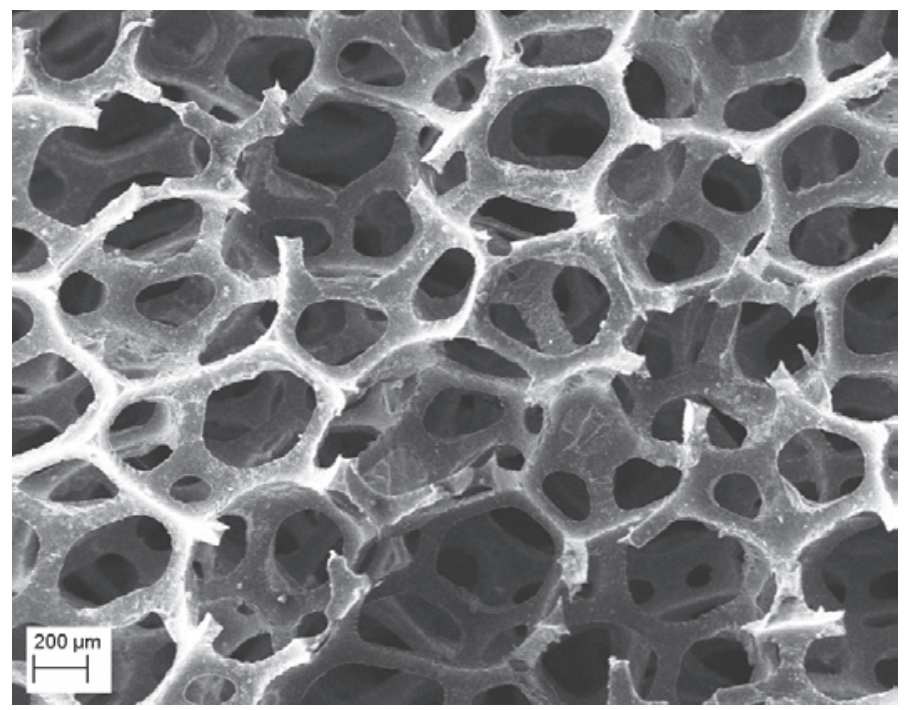

Fig. 1. Structure of resistive material on the basis of polyurethane foam. 
Amount of graphite should be enough to ensure the percolation threshold. To give the properties of non-flammable material is processed with a special compound does not influence the radio-technical characteristics. Carbon particles have sizes considerably less than the wavelength and skin layer thickness, and form a continuous quasi-graphite conductive mesh. The final DC conductivity and all the features in the microwave range depend on the amount of carbon filler, the structure, thickness and length of the conducting clusters. Such material can be considered as uniform on the electrical properties (although this may be the subject of a separate study), and isotropic.

Figure 2 presents the electron microscopy of conductive graphite coating of various types. It was determined that smaller particles of graphite form more smooth continuous film on the surface of the pores of the polymer: top left - "rough" film formed by the coarse fraction of graphite particles, bottom left - "smooth" film formed by fines. A "slice" of a graphite particle is presented there, it is seen that thickness $h$ is about 26 interlayer distances, hence $h=26 x$ $0.335 \approx 9 \mathrm{~nm}$. However, the average transverse size of the graphite particles is much greater than the thickness - size distribution is shown in Figure 3. Consequently, the particles are disk-shaped with a large aspect ratio. This is confirmed by data of X-ray diffraction analysis, namely, according to the size of the region of the coherent scattering, determined smaller of the sizes (Prokof'ev et al., 2010). This form with the condition of surface area contributes to shaping of the steady conductive coating.
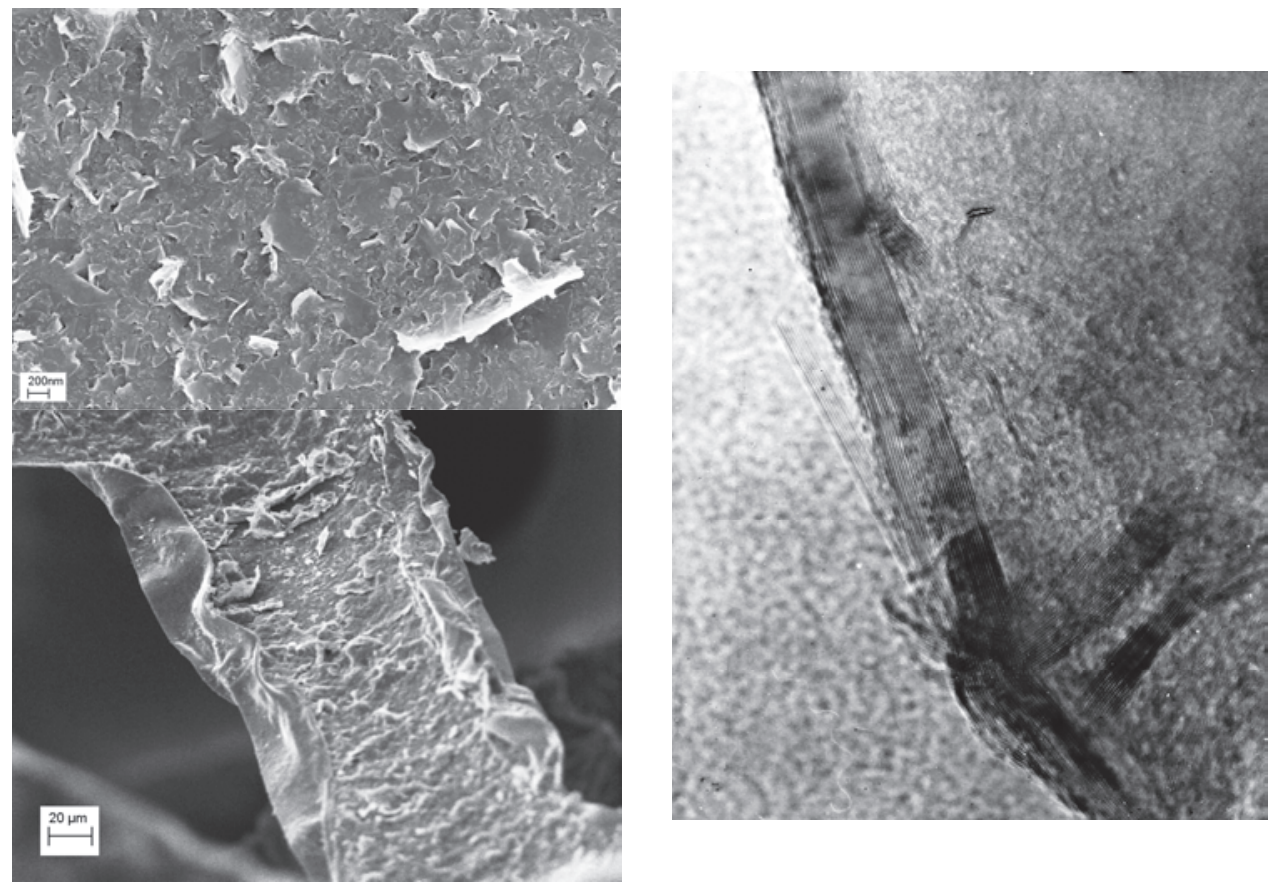

Fig. 2. Electron microscopy of conductive graphite coating of various types. Top left "rough" film formed by the coarse fraction of graphite particles. Bottom left - "smooth" film formed by fines. Right - the "slice" of the graphite particles with thickness of about 26 interlayer distances. 
With the creation of composite materials for absorbing or shielding electromagnetic radiation of radiofrequency range, are effective the composites, which possess a certain resistivity in DC regime, i.e., with the concentration of the filler $p \geq p_{\mathrm{c}}$ where $p_{\mathrm{c}}$ is the percolation threshold. When optimizing the parameters of such materials is often considering that the material is completely characterized at macro scope level in two parameters: scalar dielectric permittivity $\varepsilon^{\prime}=\operatorname{Re}(\varepsilon)$ and the specific volume electrical conductivity $\sigma_{\mathrm{V}}$, independent of frequency in the studied range of frequencies. In some cases these assumptions are justified, because the conductivity of the medium in the radio frequency $\sigma(\omega)$ negligible differs from that of the DC conductivity $\sigma_{0}$, while the imaginary part of dielectric constant depends on frequency according to $\varepsilon^{\prime \prime} \sim \omega^{-1}$ (Landau \& Lifshitz, 1984 ). However, in some cases the condition $\sigma(\omega) \approx \sigma_{0}$ is not observed exactly. At the same time, during the construction of the flat multilayer constructions, where the resonance wave effects are completely important, it is necessary to know the frequency dependence (dispersion) of the generalized dielectric constant: $\varepsilon^{*}(\omega)=\varepsilon^{\prime}(\omega)-i \cdot \varepsilon^{\prime \prime}(\omega)$.

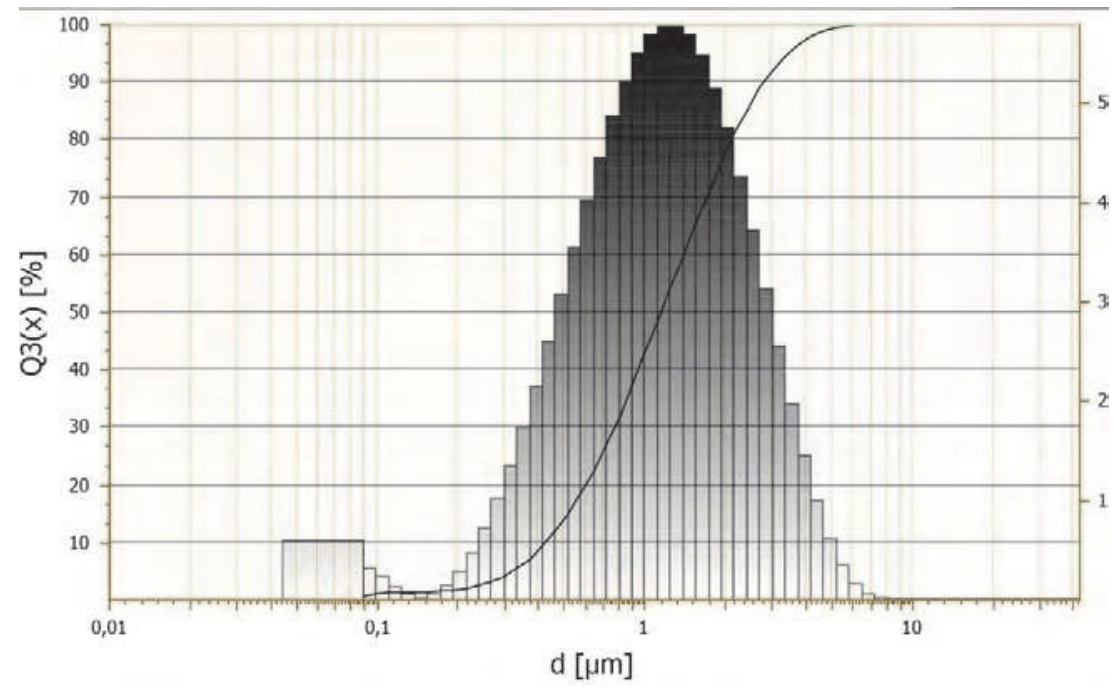

Fig. 3. Histogram of the transverse size of the colloidal graphite particles (according to photodynamic scattering).

Furthermore, often it is necessary to determine the function of the frequency dependence of dielectric constant for the correct extrapolation of experimental data into the extended frequency domain. Thus, for instance, the frequency spectrum of many UWB devices, in particular, geo-radars, stretches into region $10^{7} \ldots 10^{10} \mathrm{~Hz}$. Characterization of conductive foam is convenient to produce contactless method in the microwave range. With the measurements of such materials in the sub-gigahertz region noncontact method requires the large sizes of irradiators and models, and contact method is characterized by the poor reproducibility of results of measurements and requires special conditions for guaranteeing the electrical contacts. Determination of the dielectric permittivity values at low frequencies is possible if you know the dispersion in the microwave range. 
The similar material was the base of the wideband microwave absorbers of "Mokh" type ("Radiostrim" Ltd). The analysis of the dielectric permeability dispersion of the microwave absorber, made on the basis of electrically conductive polyurethane foam, is resulted below. In order to obtain the data about the frequency dependence and of materials, there were prepared flat models and in the free space the amplitude and phase responses of radiophysics coefficients were determined (for example, transmission and reflection coefficients). Then, after solving the inverse problem, which connects the experimental parameters with the dielectric constant of material at each frequency point, the frequency dispersion $\varepsilon$ (complex value) was determined.

Fig. 4 (solid curves) shows the experimental plots $\varepsilon^{\prime}$ and $\varepsilon^{\prime \prime}$, obtained from the measured values of the complex transmission coefficient for a flat sheet in an free space. Samples differ each from another by the conditions of formation of graphite coating on the surface of the pores of the polymer (a series of 3 specimens with smooth and rough coated). In each series, the fraction of graphite phase in the composite decreases from sample 1 to sample 3 $\left(\mathrm{C}_{1}>\mathrm{C}_{2}>\mathrm{C}_{3}\right)$.

It is logical to describe The structure by a polarization model like modified Maxwell-Wagner model of an inhomogeneous medium:

$$
\varepsilon^{*}=\varepsilon_{\infty}+\frac{i \cdot \sigma_{\mathrm{V}}}{\omega}+\frac{\varepsilon_{\mathrm{c}}-\varepsilon_{\infty}}{1+(j \omega \tau)^{1-\alpha}},
$$

where $\sigma_{\mathrm{v}}$ is the conductivity in DC regime, while $\varepsilon_{\mathrm{c}}, \varepsilon_{\infty} \tau$ and $\alpha$ - the same parameters as for formula (14). The term that considers through conductivity is here added, since the threshold of percolation is passed. After decomposing (15) to the real and imaginary parts, it is possible to find the parameters of the model. Fig.4 shows the curves of the real $\varepsilon^{\prime}$ and imaginary $\varepsilon^{\prime \prime}$ parts of the dielectric permittivity, obtained both experimentally (solid line) and from the calculation by formula (15) with the parameters: $\tau, \alpha, \sigma_{\mathrm{V}}, \varepsilon_{\mathrm{c}}$ and $\varepsilon_{\infty}$ (dotted line). Values of the parameters are given in Table 1 .

At $\alpha \approx 0.15 \div 0.17$ theoretically constructed curves simultaneously well coincide with experimental values $\varepsilon^{\prime}$ and $\varepsilon^{\prime \prime}$.

The analysis of the frequency dependences (see Table 1) shows that for the investigated samples the last term in expression (15) is more significant for the "rough" graphite covering, in contrast to the case of "smooth" covering, where value of $\sigma_{\mathrm{v}}$ correlates with the concentration of the graphite phase and influence of the DC-conductivity is more.

To obtain the effective microwave absorber with resistive losses is necessary to ensure optimal complex conductivity of the material in a given frequency range: it is necessary, on the one hand, efficiently convert electromagnetic energy into heat, but on the other hand - to avoid unwanted reflections from conductive surfaces (Bibikov et al., 2008). Usually for this purpose are used the structures of gradient-type material, or profiles, minimizing reflection from the front edge of the absorber (Fig.5).

\section{Composite materials based on ferromagnetic fillers}

Besides the microwave absorber possessing dielectric losses, the composite materials, which contain ferromagnetic phase, are used. Special features and conditions of using such materials it will be discussed further. 


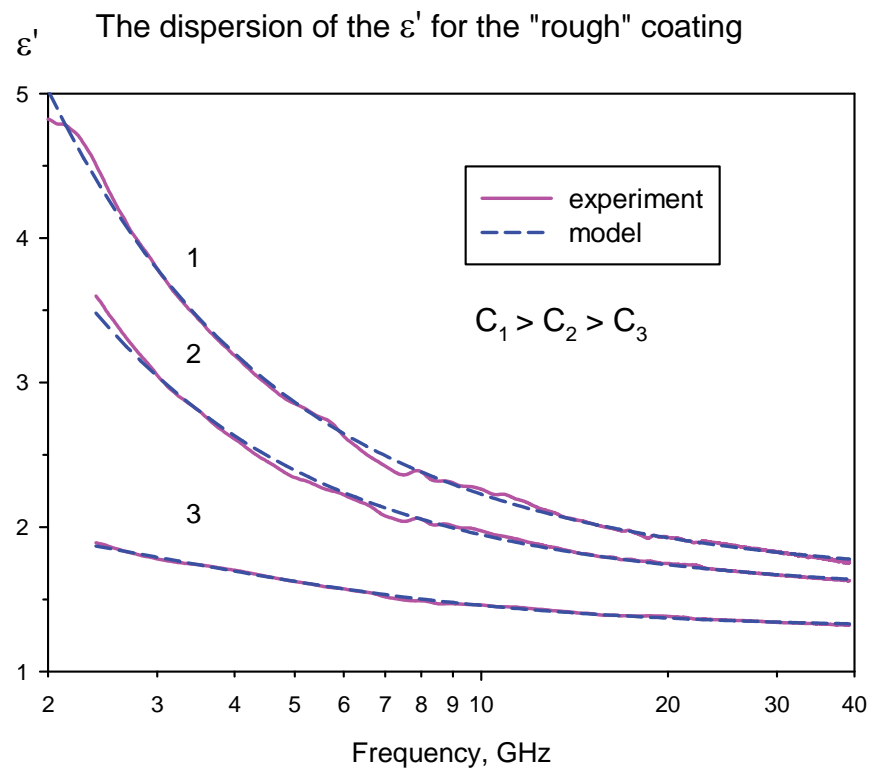

(a)

$\varepsilon " \quad$ The dispersion of the $\varepsilon$ " for the "smooth" coating

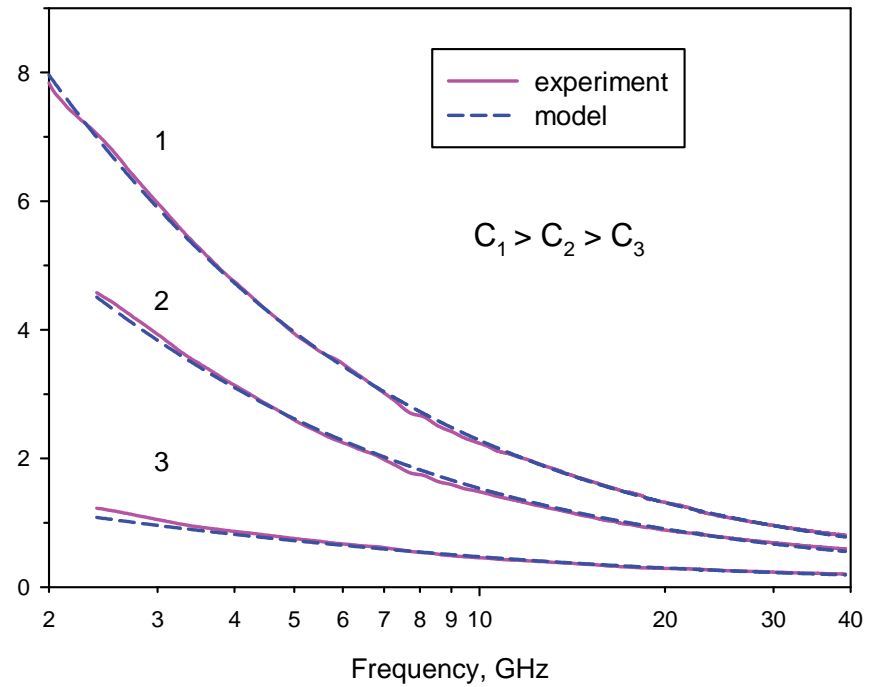

(b)

Fig. 4. Frequency dependence of dielectric permittivity of the conducting foamed PPU with the "smooth" covering: (a) $-\varepsilon^{\prime}$, (b) $-\varepsilon^{\prime \prime}$. 
$\varepsilon^{\prime} \quad$ The dispersion of the $\varepsilon^{\prime}$ for the "smooth" coating

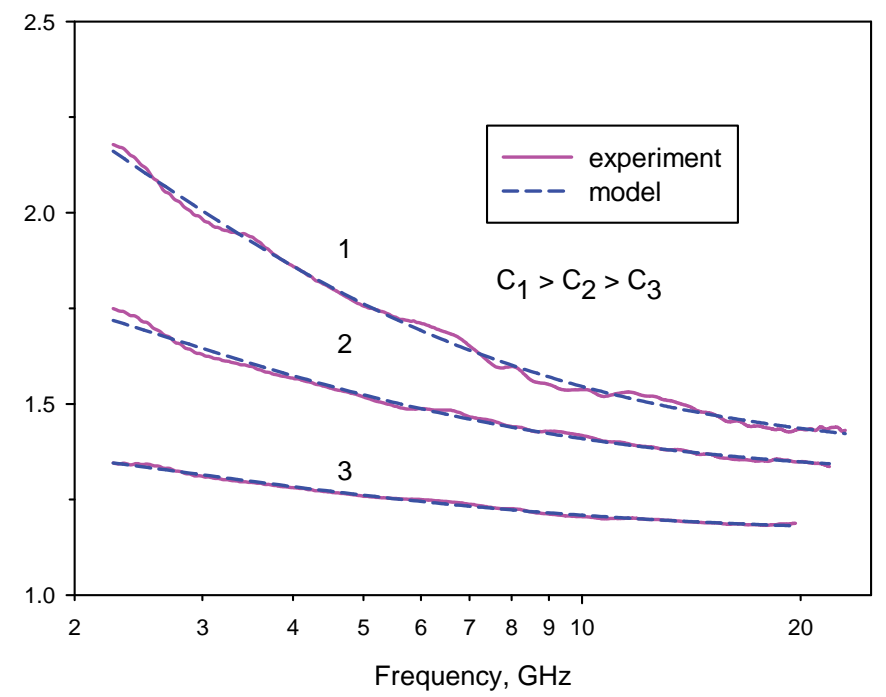

(a)

$\varepsilon$ " The dispersion of the $\varepsilon$ " for the "smooth" coating

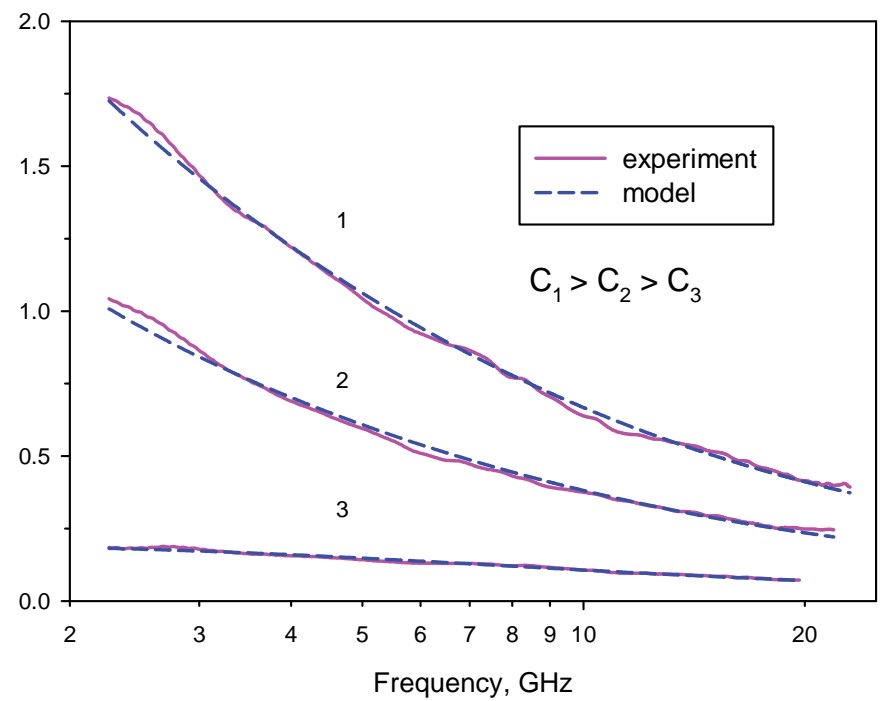

(b)

Fig. 5. Frequency dependence of dielectric permittivity of the conducting foamed PPU with the "rough" covering: (a) $-\varepsilon^{\prime},(\mathrm{b})-\varepsilon^{\prime \prime}$. 


\begin{tabular}{|c|c|c|c|c|c|c|}
\hline Type & Sample & $\tau, \mathrm{ns}$ & $\sigma_{\mathrm{V}}, \Omega^{-1} \mathrm{~m}^{-1}$ & $\Delta \varepsilon$ & $\varepsilon_{\infty}$ & $1-\alpha$ \\
\hline \multirow{3}{*}{ "smooth" } & 1 & 0.47 & 0.126 & 1.73 & 1.33 & 0.83 \\
\cline { 2 - 7 } & 2 & 0.39 & 0.084 & 0.79 & 1.29 & 0.83 \\
\cline { 2 - 7 } & 3 & 0.36 & 0.006 & 0.33 & 1.15 & 0.83 \\
\hline \multirow{3}{*}{ "rough" } & 1 & 2.91 & 0.07 & 38.5 & 1.61 & 0.85 \\
\cline { 2 - 7 } & 2 & 2.26 & 0.05 & 20.3 & 1.52 & 0.85 \\
\cline { 2 - 7 } & 3 & 0.33 & 0.09 & 0.99 & 1.29 & 0.85 \\
\hline
\end{tabular}

Table 1. Dispersion parameters of composites based on polyurethane foam with the graphite covering.

\subsection{The motivation of the using of ferromagnetic phase in composite materials}

One problem is the unwanted reflections from the boundary of the absorbing material. A way to reduce the reflection coefficient of the interface is the impedance matching material and free space. It is due to the mismatching of $\mu / \varepsilon$ ratio at the interface between free space and the edge of the material. Since the mismatch is due to the high complex permittivity, proportional increasing of the complex permeability can compensate it.

To ensure electromagnetic compatibility and testing a number of devices, for example, communication, UWB devices (georadar etc.), and for equipment of proper anechoic chambers, it is necessary to use radio shielding and absorbing materials of a range of frequencies from $\mathrm{MHz}$ up to $\mathrm{GHz}$. Because of the geometric limitations, absorbers of resistive type are unacceptable here; therefore it is necessary to use materials with the high magnetic losses. For example, it possible to use the materials on the basis of ferrites or magnetic metals, which are characterized by an increase in the losses with the resonance of domain walls in the $\mathrm{MHz}$ region, particularly due to the special structure of the ferrite grains (Bibikov et al., 2010).

\subsection{Ferrite-based composites}

Analysis of the properties of ferrites at microwave frequencies shows that as an absorber of irradiation in the long wavelength part of the microwave range is preferable to use $\mathrm{Mn}-\mathrm{Zn}$ ferrites, for UHF region - Ni-Zn ferrites, in the centimeter wavelength region - hexagonal ferrites. Using the high-anisotropic hexagonal ferrites as fillers of composite materials allows realizing the frequency-selective absorption of electromagnetic radiation by controlling the frequency of natural ferromagnetic resonance of the ferrites.

But the use of ferrite ceramics is often inconvenient due to specific technological and operational requirements for many electronics products. The composite material consisting of an insulating matrix and ferromagnetic filler called magnetodielectrics. As the fillers of magnetic dielectrics frequently are used carbonyl iron, Alsifer, Permalloy, etc. Magnetic permeability of magnetic dielectrics is less than in monolithic ferromagnetic materials for two reasons: in first, the presence of the significant demagnetizing factor because of the dissociation of particles and, in second, the formation of the poly-domain structure is energetically disadvantageous. In spite of it, the magnetic composite materials possess sufficient magnetic losses for the using as microwave absorbers. For example, Fig. 6 shows the reflection coefficient of the composite material based on granular Ni-Zn ferrite at a mass filling of $89 \%$. 


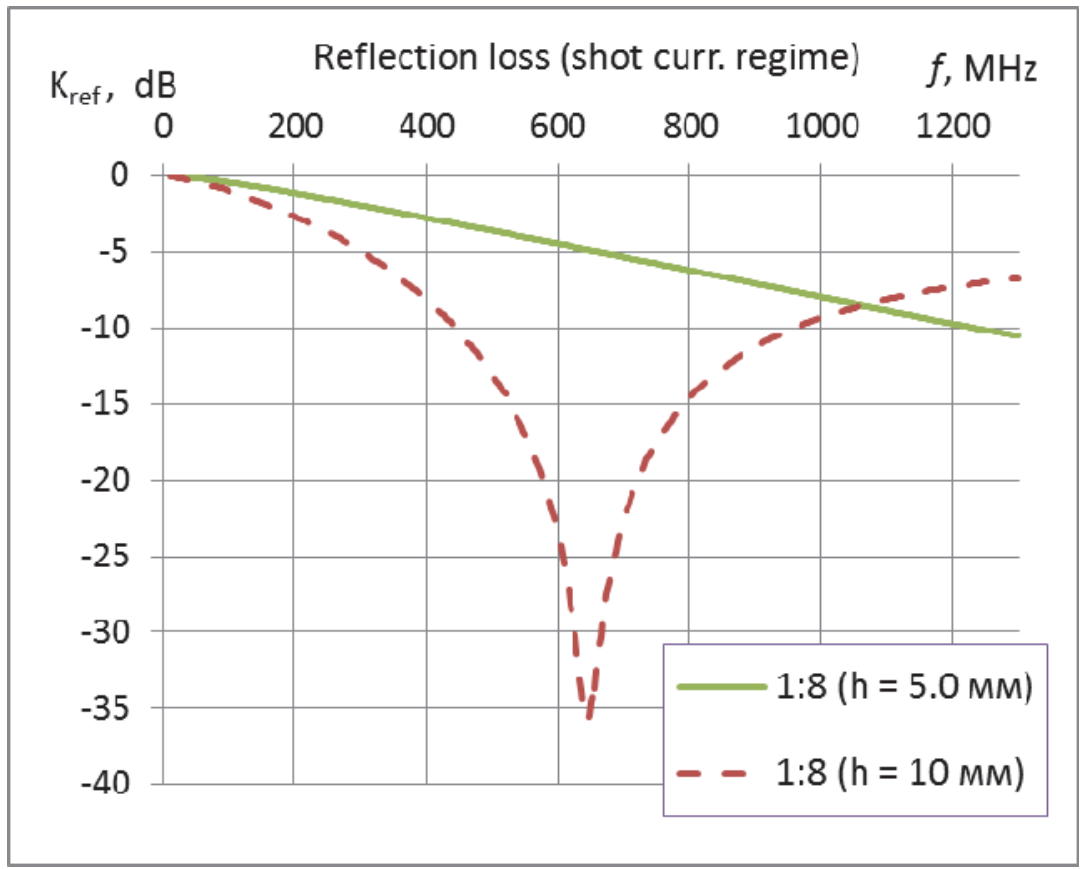

Fig. 6. The reflection coefficient of the composite materials on the basis of Ni-Zn ferrite. (Two thicknesses of the samples).

\subsection{The composites based on amorphous magnetic alloys.}

Ferrite-polymer composites present narrow-band electromagnetic wave absorbers for use in several hundred megahertz to several gigahertz frequency range. The merits of ferritepolymer composite absorbers are their adequate reflection loss due to large magnetic loss. However, the major limitation for expanding the application of ferrite-polymer composites is that possesses quite different frequency dispersion of the dielectric permittivity $\varepsilon$ and magnetic permeability $\mu$. This limits the working frequency range of the material. The development of new thinner electromagnetic wave absorbers using amorphous alloys has been attempted in order to overcome the ferrite-polymer composites problem.

Since the permeability of amorphous alloys rapidly decreases with increasing frequency due to eddy current loss, composites which are fabricated by dispersing amorphous alloy particles in a polymer matrix are generally used in high frequency applications. However, the application of amorphous alloy composites as absorbers is restricted by large surface reflection of electromagnetic wave due to their relatively large complex permittivity values compared to complex permeability values.

Then, it is possible to smoothly change magnetic permeability, controlling by the restructuring of magnetic alloy from the amorphous into the nano-crystalline state.

The next sample of composite material consists of organosilicon thermo-stable matrix and mixed nanocrystal-amorphous magnetic filler with grain size near $50 \mu \mathrm{m}$ (Kuznetsov et al., 2005; Bibikov et al., 2007). 


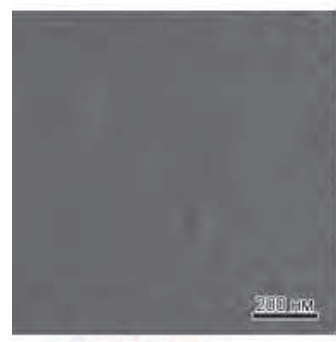

Initial state

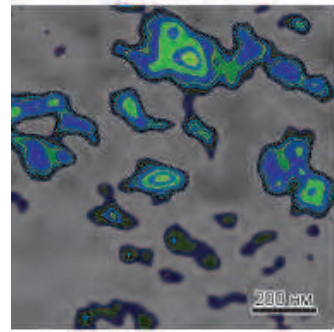

$470{ }^{\circ} \mathrm{C}$

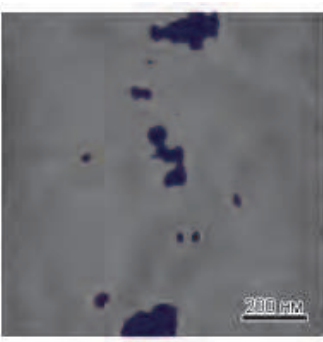

$430^{\circ} \mathrm{C}$

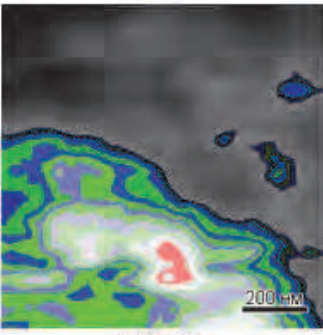

$510^{\circ} \mathrm{C}$

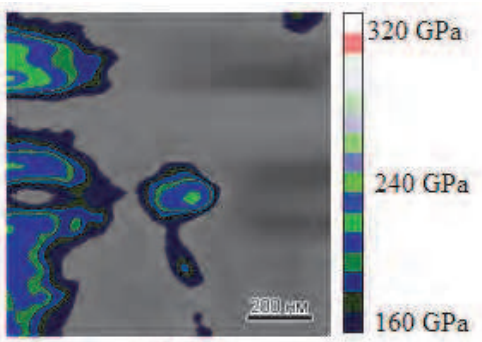

$450^{\circ} \mathrm{C}$
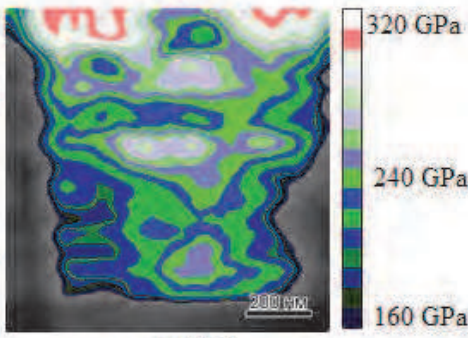

$550^{\circ} \mathrm{C}$

Fig. 7. Atomic force microscopy:Fe-Cu-Si-Nb-B alloy elastic modulus via temperature of the alloy treatment

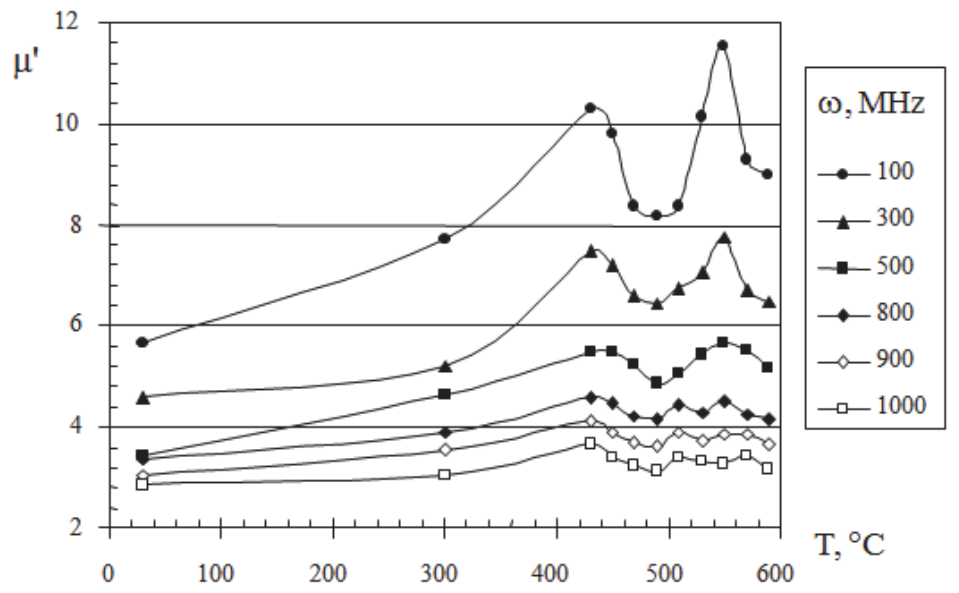

Fig. 8. Dependence of the permeability $\mu^{\prime}$ at $f=100 \ldots 1000 \mathrm{MHz}$ on the temperature treatment of the amorphous magnetic material. (Content of the magnetic powder $\sim 50 \%$ vol.)

Using the microscope of atomic forces, it was shown (Fig.7) that a process of formation of nanocrystalline areas in the amorphous matrix takes place during mechanical and thermo treatment of the alloy. This is confirmed by the pictures of the micro-distribution of the 
modulus of elasticity. At the same time, it was observed the correlation between growth of the nano-crystalline regions in the filler and the increasing of the effective magnetic permeability of composite (Fig.8). This leads to noticeable changes integral radiophysical characteristics (Fig. 9).
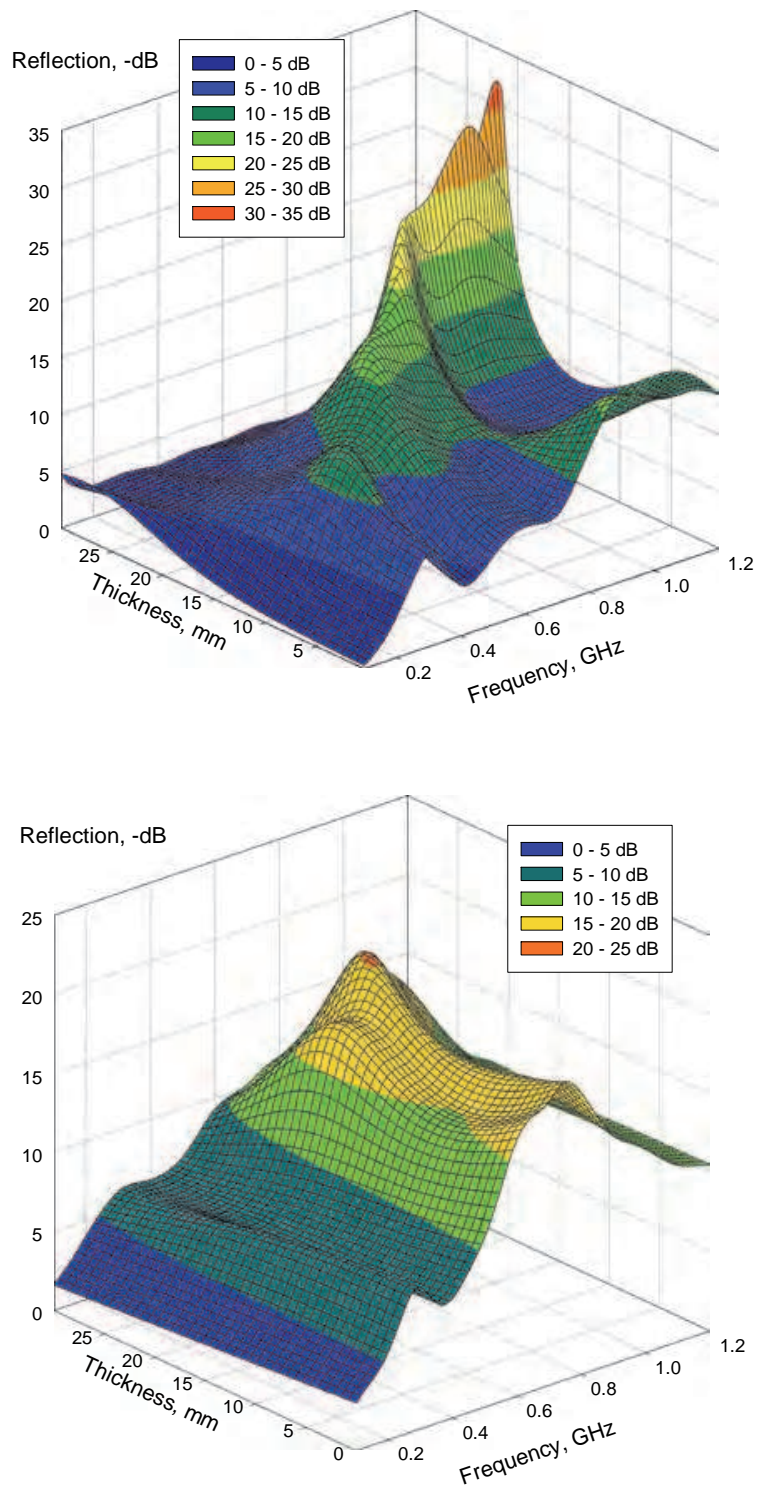

Fig. 9. The dependence of the reflection from the thick-layer coatings based on experimental data of the composite filled with amorphous crystalline alloy. Top - the filler in the amorphous state, bottom - in nanocrystalline state. 


\section{The possibility of the using of nanostructured composites for radiophysics applications}

A number of theoretical works according to the electromagnetic and of carbonic nano-tubes shows that for further progress in the field of creating the thin-film effective microwave absorbers it is necessary to investigate the influence of magnetic nanoparticles in the insulating and semiconductor tape matrices. Also, the granular structures containing ferromagnetic nanoparticles of 3-d metals in such matrices can have a high degree of microwave absorption. It is defined by a set of factors: a large magnetization and the rapid relaxation of the spin of nanoparticles are in a superparamagnetic state, as well as a large value of dielectric constant and dielectric loss, depending on the localized electronic states in the matrix. Thus, several studies have investigated the film hydrogenated amorphous carbon nanoparticles Cm deposited on policar and Kevlar substrate (Rozanov et al., 2005).

\section{Microwave absorbers based on multilayered, gradient and profiled composite materials}

The absorbers of resistance type microwave emission can effectively work in entire wavelength range of radio-frequency range, but, taking into account reduction in the effectiveness with an increase in the relation of $\lambda / h$, where $\lambda$ - wavelength and $h$ - thickness of the material, they are frequently used at frequencies higher $1 \mathrm{GHz}$. . Of course, it is possible to use this material at frequencies below $1 \mathrm{GHz}$, but the size of it should be much greater (proportionally to the wavelength $\lambda$ ).

An example of pyramidal microwave absorber is on Fig.10. The black color is due to the carbon-graphite filler, and white color - after processing by incombustible agent. Frequency dependence of the reflectivity of the samples of composite materials such as moss of various heights are shown in Fig. 11 (Bibikov et al., 2010). The graphs in Fig.11 show that the pyramidal structure based on the PPU with fillers discussed above, provides a significant reduction of electromagnetic radiation incident along the normal. So, for the absorber, "Mokh P-330" with a height of $330 \mathrm{~mm}$ reflected microwave power is less than $50 \mathrm{~dB}$, which is equivalent to the attenuation of the power at 10,000 times, and with the decreasing in the height of the material the reflection coefficient increases at low frequencies - for most thin absorber "Moss P-70" the interference is observed.

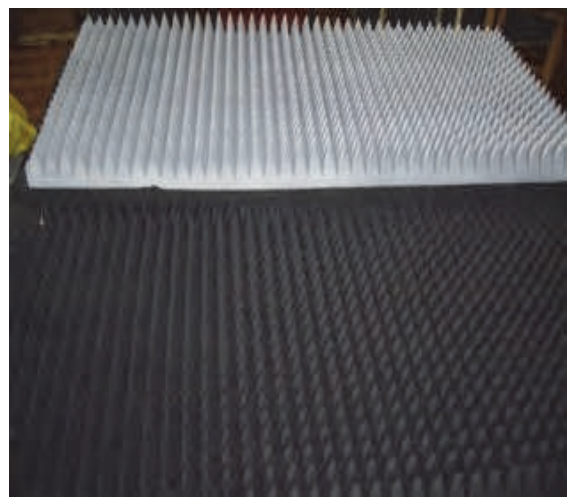

Fig. 10. View of microwave absorber "Mokh P-70". 


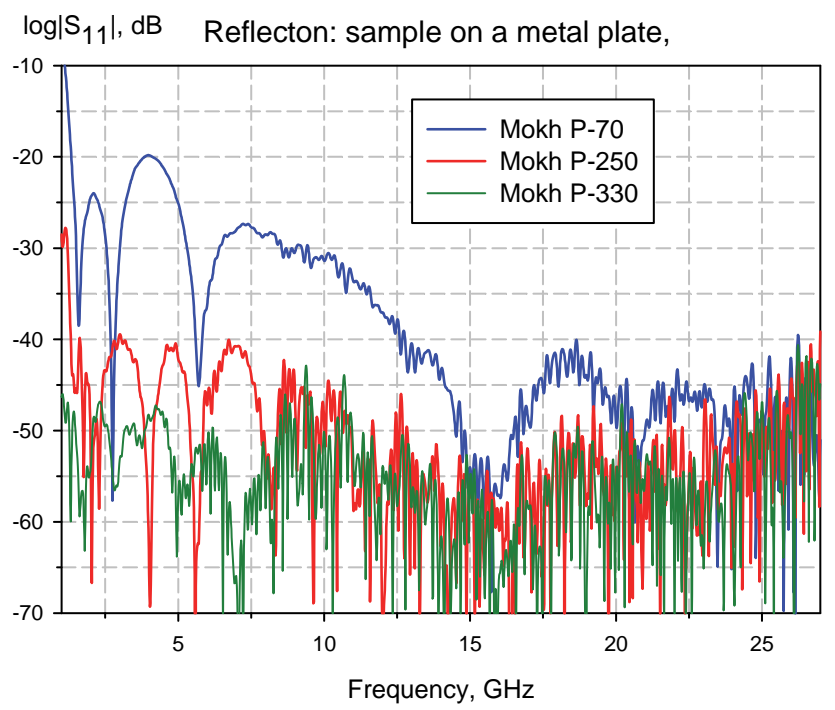

Fig. 11. Coefficient of reflection of the electromagnetic irradiation from the microwave absorber "Mokh P-70" at normal incidence in free space.

This type of material is useful both for the equipment of anechoic chambers, and for electromagnetic compatibility of radio systems (communication, radar, medical and search equipment, etc.).

\section{Conclusion}

The using of composite materials with desired radiophysical properties in some cases is optimal solution for the complex engineering problems in modern electronics. In particular, these are:

- Ensuring the electromagnetic compatibility of electronic equipment;

- Information security,

- Electromagnetic security and the environment safety,

- Functionality of radio systems: communications equipment, radars, control system, ultra-wideband devices, etc.

- Tasks of Military and special techniques.

Special feature of the composite materials for radiotechniques is the correspondence simultaneously electrophysical, mechanical, and sometimes also to the chemical parameters. The complexity of creating a composite broadband microwave absorbers determined by the fact that the values of complex permittivity, permeability and, correspondingly, the coefficient of reflection of radio waves depends on the frequency of the electromagnetic field.

The ideal absorber of the electromagnetic radiation of radio-frequency s-band has not been created. That's why design of the similar materials is always defined by a number of requirements both for radiophysics and operational parameters of the material. Therefore there are different types of absorbers, including on the basis of composite materials: of resistive type, ferromagnetic, gradient type, shaped, etc. 


\section{References}

Bibikov S.B., Kulikovsky Ed.I., Sharafiev R.S. \& Kuznetsov P.A. (2007). New absorbing materials for applications in decimeter and millimeter wavelengths ranges, Proceedings of the International Conference on Antenna Theory and Techniques, pp. 295297, ISSN 978-1-4244-1578-7, Sevastopol, Ukraine, September, 2007.

Bibikov S.B., Gorshenev V.N., Sharafiev R.S. \& Kuznetsov A.M. (2008). Electrophysical properties of electroconducting papers and cardboards treated with colloidgraphite solutions Materials Chemistry and Physics. Vol. 108, No.1, pp. 39 - 44, ISSN 0254-0584.

Bibikov S.B., Smolnikova O.N., Prokof'ev M.V., Orlov V.V. \& Men'shova S.B. (2010). Some trends in design of microwave absorbers for anechoic chambers and ultrawideband systems. Proceedings of the 5th International Conference on Ultrawideband and Ultrashort Impulse Signals, pp. 262-265, ISBN 978-1-4244-7468-4, Sevastopol, Ukraine, September, 2010.

Bibikov S.B., Smolnikova O.N. \& Prokof'ev M.V. (2011). Dielectric properties and the microwave - conductivity of porous radioabsorbing materials. Radiotekhnika (Radioengineering), No.3, Will be published (March 2011), ISSN 0033-8486.

Efros A.L. \& Shklovskii B.I. (1976). Critical Behavior of Conductivity and Dielectric Constant near the Metal-Non-Metal Transition Threshold, Phys.Stat.Sol.(B), Vol. 76, No2, pp. 475-785.

Gorbatenko O.N. \& Bibikov S.B. (2006). The using of radar absorbing - radio scaterring for protection against electromagnetic interference of georadar. Special Technics, No. 3, pp.26. ISSN 1996-0506.

Gorshenev V.N., Bibikov S.B., Spector V.N. (1997). Simulation, synthesis and investigation of microwave absorbing composite materials, Synthetic Metals, Vol. 86, pp. 22552256, ISSN 0379-6779.

Kirkpatrick S. (1973). Percolation and Conduction. Rev.Mod.Phys.Vol. 45, No. 4, pp. 574-588.

Kuznetsov P.A., Zvorygin R.G. \& Bibikov S.B. (2005). Investigation of atomic-force microscope crystallization kinetics of the nanocrystalline alloy $\mathrm{Fe}-\mathrm{Cu}-\mathrm{Nb}-\mathrm{Si}-\mathrm{B}$ and the creation of systems based on its electromagnetic protection. Metally (Metals), 2005, No 6, pp. 25-31, ISSN 0869-5733.

Lutsev L.V., Nikolaychuk G.A., Petrov V.V., Yakovlev S.V. (2008). Multi-purpose radioabsorbing materials based on magnetic nanostructures: preparation, properties and applications. Nanotechnics, No.14, pp.37-43, ISSN 1816-4498.

Lagarkov A.N. \& Sarychev A.K. (1996). Phys. Rev. B, Vol.53, No.7, (March 1996), pp. 63186336, ISSN 0163-1829.

Landau L.D. \& Lifshitz E.M. (1984). Electrodynamics of Continuous Media, 2th ed., Pergamon, Oxford.

Maksimenko S.A., Slepian G.Ya. (2002). Electrodynamics of carbonic nanotubes. Radioelectronics and Communications Systems Vol. 47, No. 3, pp. 261-280, ISSN 0033-8494.

Prokof'ev M.V., Smolnikova O.N., Bibikov S.B. \& Kuznetsov A.M. (2010). Influence of conditions of heat treatment on the structure and properties of the coatings from colloidal-graphite dispersions. Aerospace MAI Journal, Vol. 17, No.2, pp.78-86, ISSN 0869-6101. 
Rozanov K.N., Li Z.W., Chen L.F. \& Koledintseva M.Y. (2005). Microwave permeability of $\mathrm{CO}_{2} \mathrm{Z}$ composites, Journal of Applied Physics, Vol.97, No.1. pp. 013905-1-7, ISSN 00218979 .

Stauffer D. \& Aharony A. (2003). Introduction to Percolation Theory (2003), London: Taylor \& Francis.

Titov A.N., Bibikov S.B. \& Kulikovskij Ed.I. (2008). Proceedings of the 4th International Conference on Ultrawideband and Ultrashort Impulse Signals, pp. 248-250, ISBN 978-14244-2738-3, Sevastopol, Ukraine, September, 2008. 


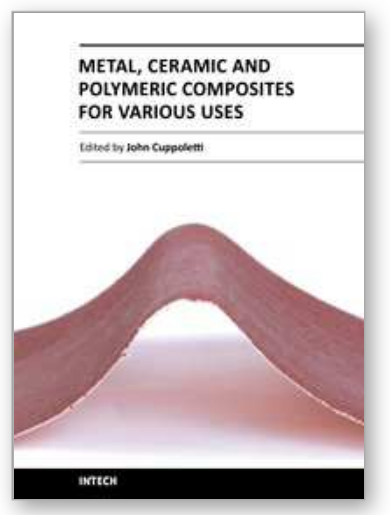

\author{
Metal, Ceramic and Polymeric Composites for Various Uses \\ Edited by Dr. John Cuppoletti
}

ISBN 978-953-307-353-8

Hard cover, 684 pages

Publisher InTech

Published online 20, July, 2011

Published in print edition July, 2011

Composite materials, often shortened to composites, are engineered or naturally occurring materials made from two or more constituent materials with significantly different physical or chemical properties which remain separate and distinct at the macroscopic or microscopic scale within the finished structure. The aim of this book is to provide comprehensive reference and text on composite materials and structures. This book will cover aspects of design, production, manufacturing, exploitation and maintenance of composite materials. The scope of the book covers scientific, technological and practical concepts concerning research, development and realization of composites.

\title{
How to reference
}

In order to correctly reference this scholarly work, feel free to copy and paste the following:

Sergey Bibikov and Mikhail Prokof'Ev (2011). Composite Materials for Some Radiophysics Applications, Metal, Ceramic and Polymeric Composites for Various Uses, Dr. John Cuppoletti (Ed.), ISBN: 978-953-307-353-8, InTech, Available from: http://www.intechopen.com/books/metal-ceramic-and-polymeric-composites-forvarious-uses/composite-materials-for-some-radiophysics-applications

\section{INTECH}

open science | open minds

\section{InTech Europe}

University Campus STeP Ri Slavka Krautzeka 83/A 51000 Rijeka, Croatia Phone: +385 (51) 770447 Fax: +385 (51) 686166 www.intechopen.com

\section{InTech China}

Unit 405, Office Block, Hotel Equatorial Shanghai No.65, Yan An Road (West), Shanghai, 200040, China 中国上海市延安西路65号上海国际贵都大饭店办公楼 405 单元 Phone: +86-21-62489820

Fax: +86-21-62489821 
(C) 2011 The Author(s). Licensee IntechOpen. This chapter is distributed under the terms of the Creative Commons Attribution-NonCommercialShareAlike-3.0 License, which permits use, distribution and reproduction for non-commercial purposes, provided the original is properly cited and derivative works building on this content are distributed under the same license. 